\title{
Molecular Marker Technology for Crop Improvement
}

\author{
Jose Miguel Soriano \\ Sustainable Field Crops Programme, IRTA (Institute for Food and Agricultural Research and Technology), \\ 25198 Lleida, Spain; josemiguel.soriano@irta.cat
}

Received: 3 September 2020; Accepted: 23 September 2020; Published: 24 September 2020

\begin{abstract}
Since the 1980s, agriculture and plant breeding have changed with the development of molecular marker technology. In recent decades, different types of molecular markers have been used for different purposes: mapping, marker-assisted selection, characterization of genetic resources, etc. These have produced effective genotyping, but the results have been costly and time-consuming, due to the small number of markers that could be tested simultaneously. Recent advances in molecular marker technologies such as the development of high-throughput genotyping platforms, genotyping by sequencing, and the release of the genome sequences of major crop plants open new possibilities for advancing crop improvement. This Special Issue collects sixteen research studies, including the application of molecular markers in eleven crop species, from the generation of linkage maps and diversity studies to the application of marker-assisted selection and genomic prediction.
\end{abstract}

Keywords: crop breeding; genetic maps; QTL mapping; GWAS; marker assisted selection; genomic selection; DNA sequencing

\section{Introduction}

Classical breeding was the main approach used by breeders to increase crop productivity during the 20th century. It implies the selection of cultivars with the desired characteristics for the target trait, usually morphological or visual characteristics. The best genotypes were selected and used as parents in a backcross scheme with a recurrent parent to dilute the irrelevant or undesired traits [1]. However, the long time to get a commercial cultivar and limitations related to traits highly dependent on the environment or with low heritability make necessary the use of complementary approaches to assist the breeding process. The development of molecular biology made possible the appearance of a new type of marker based on polymorphisms in the DNA sequence, the molecular markers, which broaden the possibilities for new challenges in plant breeding. Molecular markers are widely distributed in the genome, they are not affected by the environment, and they can be identified in any tissue and developmental stage. From their development, their use in agriculture increased through the construction of genetic maps in crop species, the association between molecular markers and important agronomic traits, the dissection of quantitative traits, and the positional cloning of genes of interest. Besides the estimation of genetic distances and molecular cloning, molecular markers provide the most suitable tool for the evaluation of genetic diversity, allowing for the selection of the most suitable parental lines in breeding programs, the management of germplasm collections, and varietal identification [2].

Once the association between a marker and a trait is detected, it can be deployed into a breeding program through marker-assisted selection (MAS). The success of this technique relies on the identification of markers tightly linked with the genome region of a target trait. MAS improves the efficiency of the selection of Mendelian traits, facilitating the introgression of single genes with the desired alleles into elite cultivars and removing the undesirable genome of the donor parent in a backcrossing program, and allows for the identification and protection of commercial cultivars 
through fingerprinting [3]. Although MAS has been effectively used before for Mendelian traits or those regulated by a low number of genes, many of the agronomic traits show a quantitative nature and are influenced by the environment [4]. In the last decade, the development of high-throughput genotyping platforms has allowed for the screening of whole genomes for the selection of desired traits. In addition, novel statistical approaches for the use of large amounts of genetic and phenotypic data have been developed. Altogether, this has allowed for the development of new selection strategies, such as the genomic prediction or selection (GS), which attempts to skip the limitations of MAS [5].

Coupled with the rapid development of high-throughput genotypic technology, the next DNA sequencing technologies and their applications in genetic and physical mapping have made significant progress in accelerating plant breeding with a lower cost.

\section{Overview of the Special Issue}

This special issue of Agronomy with the title "Molecular marker technology for crop improvement" publishes 16 articles providing insights into the different applications of molecular markers in plant breeding. Eleven crop species were analyzed with six different approaches.

Although the development of linkage maps is the first step for gene identification and many maps have been developed in several crop species, novel marker approaches involving high-throughput genotyping are evolving for the construction of highly saturated maps. A high-density single nucleotide polymorphism (SNP) linkage map in potato was developed using a recently developed strategy for the discovery of SNPs, the specific length amplified fragment sequencing (SLAF-seq) approach [6].

Biparental quantitative trait loci (QTL) mapping is a classical approach to identify multi-genic traits. The success of detecting a QTL depends on several factors: (1) marker density, (2) population size, and (3) trait heritability. As a classical approach in crop breeding, within the Special Issue it was applied for the identification of the loci controlling milling yield in rice [7], resistance to Striga hermonthica in maize [8], and leaf rust and stem rust resistance in wheat [9]. These studies identified new loci for important traits in breeding and will be the starting point for a deeper analysis of candidate gene identification.

Genome-wide association studies (GWASs) have become a valuable tool in recent years as a complementary approach to biparental mapping, providing broader allelic coverage and higher mapping resolution. In this issue, GWASs were performed for the analysis of seminal roots in landraces of wheat and durum wheat from the Mediterranean basin [10,11], agronomic and quality traits in elite durum wheat [12], and for flowering time in maize inbred lines [13]. The studies of Roselló et al. [10] and Rufo et al. [11] pointed out the usefulness of the old germplasm to be used as genetic resources for improving drought-related traits in the breeding programs to broaden the genetic variability. Merida-García et al. [12] combined a GWAS with a candidate gene approach to successfully identify gene clusters involved in important traits for wheat breeding. The study of Maldonado et al. [13] revealed that the use of a GWAS based on haplotype blocks was more efficient than the standard approach to identify major effect loci, and the network-assisted gene prioritization used identified four genes influencing flowering time in tropical maize.

Genetic diversity is crucial for crop improvement, as it allow breeders to identify appropriate parents to be included in breeding programs for broadening genetic variability. Within this Special Issue, the genetic diversity of wheat, avocado, and raspberry was assessed by high-resolution melting (HRM), and insertion site-based polymorphism (ISBP) markers, simple sequence repeats (SSR) developed from single-molecule long-read sequences, and SSRs from flavonoid biosynthesis genes, respectively [14-16]. Merida-Garcia et al. [14] developed ISBP markers for the wheat genome as an alternative to SSRs and SNPs. The authors concluded that these HRM-ISBPs are a cost-effective and efficient marker approach for wheat breeding programs, being also useful for gene tagging. The studies of Ge et al. [15] and Lebedev et al. [16] demonstrated the power of SSR markers. Although they were developed three decades ago [17], they are still commonly used because of their codominant and multi-allelic nature and high reproducibility. 
The abovementioned studies in this Special Issue have taken into account the use of molecular markers for the development of linkage maps, the analysis of genetic diversity, and the mapping of quantitative traits, by means of the classic biparental QTL mapping or GWASs. The rest of the studies represent direct applications of molecular markers after these previous steps of development, location in a linkage map, and genotype-phenotype association: MAS and GS.

Marker-assisted selection was applied in four studies for (1) the selection of parental germplasm in sugarcane breeding programs [18], (2) improving blast resistance and salt tolerance in rice [19], (3) the selection of the pollination constant non-astringent (PCNA) type in Spanish germplasms of persimmon [20], and (4) the selection of resistance to plum pox virus (PPV) in apricot by allele-specific PCR [21]. Wu et al. [18] identified two groups among the 150 most widely used sugarcane parental clones. Based on these results, the authors could identify the most appropriate cultivars to broaden the genetic base of breeding germplasm. Thanasilungura et al. [19] improved the rice cultivar RD6 for salt tolerance with the QTL "Saltol" and blast resistance with four different QTLs by marker-assisted backcrossing and phenotypic selection. The authors found that one of the introgression lines showed superior salt tolerance and blast resistance, maintaining higher quality and agronomic performance than RD6. In the study of the selection of the PCNA type of persimmon, Blasco et al. [20] identified in the Spanish germplasm of persimmon the previously developed markers DISx-AF4, linked to the production of male flowers, and AST, linked to fruit astringency. The screening of these markers in different progenies of backcrosses demonstrated a very low rate of selection of both traits together and is thus a valuable tool in a breeding program. The last example of MAS in this Special Issue corresponds to the selection of PPV resistance in the apricot breeding program at IVIA (Valencia, Spain) [21]. In this study, the authors present a high-throughput method for a rapid test of PPV resistance, thus improving the efficiency of apricot breeding programs at a low cost. MAS is of special interest in fruit trees due to the long time needed to obtain a new generation.

Finally, GS was used to estimate the breeding values for grain composition in sorghum [22]. Although GS was developed initially for animal breeding, its use in plant breeding has been extended in recent years. GS emerged as a valuable tool for improving complex traits controlled by QTLs with small effects. Together with high-throughput phenotyping techniques, it has brought a revolution in breeding by enhancing the accuracy level of selection. Sapkota et al. [22] report the use of GS for grain compositional traits. The authors found that the prediction accuracy for single trait prediction was moderate to high in respect to the phenotypic measurements obtained from near infra-red spectroscopy (NIRS) prediction.

\section{Concluding Remarks}

The Special Issue covers the use of different types of molecular markers, from SSR markers developed in 1989 to the newest high-throughput marker technology, as well as different approaches for genetic mapping and the use of molecular markers to assist crop breeding as a single marker with MAS or at genome level with GS.

To meet the needs of a growing world population, crop yields must be increased under the climate change scenario predicted for the coming decades and the threat of the emergence of new pathogens. However, the information obtained by genome sequencing and its availability at low cost, the continuous development of new molecular markers, the implementation of high-throughput phenotyping tools, and speed breeding techniques will make it possible to face these new challenges. Although not directly related with the molecular marker technology of this Special Issue, the new systems based on gene editing will play an important role in the future of agriculture.

Funding: This research received no external funding.

Conflicts of Interest: The author declares no conflict of interest. 


\section{References}

1. Hou, H.; Atlihan, N.; Lu, Z.X. New biotechnology enhances the application of cisgenesis in plant breeding. Front. Plant Sci. 2014, 5, 389. [CrossRef]

2. Phougat, D.; Panwar, I.; Puina, M.; Sethi, S. Microsatellite markers based characterization in advance breeding lines and cultivars of bread wheat. J. Environ. Biol. 2018, 39, 339-346. [CrossRef]

3. Shimelis, H.; Laing, M. Timelines in conventional crop improvement: Pre-breeding and breeding procedures. Aust. J. Crop Sci. 2012, 6, 1542-1549.

4. Goutam, U.; Kukreja, S.; Tiwari, R.; Chaudhury, A.; Gupta, R.K.; Dholakia, B.B.; Yadav, R. Biotechnological approaches for grain quality improvement in wheat: Present status and future possibilities. Aust. J. Crop Sci. 2013, 7, 469-483.

5. Talukder, S.K.; Saha, M.C. Toward Genomics-Based Breeding in C3 Cool-Season Perennial Grasses. Front. Plant Sci. 2017, 8, 1317. [CrossRef]

6. Yu, X.; Zhang, M.; Yu, Z.; Yang, D.; Li, J.; Wu, G.; Li, J. An SNP-Based High-Density Genetic Linkage Map for Tetraploid Potato Using Specific Length Amplified Fragment Sequencing (SLAF-Seq) Technology. Agronomy 2020, 10, 114. [CrossRef]

7. Zhang, H.; Zhu, Y.J.; Zhu, A.D.; Fan, Y.Y.; Huang, T.X.; Zhang, J.F.; Xie, H.A.; Zhuang, J.Y. Identification and Verification of Quantitative Trait Loci Affecting Milling Yield of Rice. Agronomy 2020, 10, 75. [CrossRef]

8. Badu-Apraku, B.; Adewale, S.; Paterne, A.; Gedil, M.; Asiedu, R. Identification of QTLs Controlling Resistance/Tolerance to Striga hermonthica in an Extra-Early Maturing Yellow Maize Population. Agronomy 2020, 10, 1168. [CrossRef]

9. Genievskaya, Y.; Abugalieva, S.; Rsaliyev, A.; Yskakova, G.; Turuspekov, Y. QTL Mapping for Seedling and Adult Plant Resistance to Leaf and Stem Rusts in Pamyati Azieva $\times$ Paragon Mapping Population of Bread Wheat. Agronomy 2020, 10, 1285. [CrossRef]

10. Roselló, M.; Royo, C.; Sanchez-Garcia, M.; Soriano, J.M. Genetic Dissection of the Seminal Root System Architecture in Mediterranean Durum Wheat Landraces by Genome-Wide Association Study. Agronomy 2019, 9, 364. [CrossRef]

11. Rufo, R.; Salvi, S.; Royo, C.; Soriano, J.M. Exploring the Genetic Architecture of Root-Related Traits in Mediterranean Bread Wheat Landraces by Genome-Wide Association Analysis. Agronomy 2020, 10, 613.

12. Mérida-García, R.; Bentley, A.R.; Gálvez, S.; Dorado, G.; Solís, I.; Ammar, K.; Hernandez, P. Mapping Agronomic and Quality Traits in Elite Durum Wheat Lines under Differing Water Regimes. Agronomy 2020, $10,144$.

13. Maldonado, C.; Mora, F.; Bertagna, F.A.B.; Kuki, M.C.; Scapim, C.A. SNP- and Haplotype-Based GWAS of Flowering-Related Traits in Maize with Network-Assisted Gene Prioritization. Agronomy 2019, 9, 725.

14. Mérida-García, R.; Gálvez, S.; Paux, E.; Dorado, G.; Pascual, L.; Giraldo, P.; Hernandez, P. High Resolution Melting and Insertion Site-Based Polymorphism Markers for Wheat Variability Analysis and Candidate Genes Selection at Drought and Heat MQTL Loci. Agronomy 2020, 10, 1294.

15. Ge, Y.; Zang, X.; Tan, L.; Wang, J.; Liu, Y.; Li, Y.; Wang, N.; Chen, D.; Zhan, R.; Ma, W. Single-Molecule Long-Read Sequencing of Avocado Generates Microsatellite Markers for Analyzing the Genetic Diversity in Avocado Germplasm. Agronomy 2019, 9, 512.

16. Lebedev, V.G.; Subbotina, N.M.; Maluchenko, O.P.; Krutovsky, K.V.; Shestibratov, K.A. Assessment of Genetic Diversity in Differently Colored Raspberry Cultivars Using SSR Markers Located in Flavonoid Biosynthesis Genes. Agronomy 2019, 9, 518.

17. Tautz, D. Hypervariability of simple sequences as a general source for polymorphic DNA markers. Nucleic Acids Res. 1989, 17, 6463-6471.

18. Wu, J.; Wang, Q.; Xie, J.; Pan, Y.-B.; Zhou, F.; Guo, Y.; Chang, H.; Xu, H.; Zhang, W.; Zhang, C.; et al. SSR Marker-Assisted Management of Parental Germplasm in Sugarcane (Saccharum spp. hybrids) Breeding Programs. Agronomy 2019, 9, 449.

19. Thanasilungura, K.; Kranto, S.; Monkham, T.; Chankaew, S.; Sanitchon, J. Improvement of a RD6 Rice Variety for Blast Resistance and Salt Tolerance through Marker-Assisted Backcrossing. Agronomy 2020, 10, 1118.

20. Blasco, M.; Gil-Muñoz, F.; Naval, M.M.; Badenes, M.L. Molecular Assisted Selection for Pollination-Constant and Non-Astringent Type without Male Flowers in Spanish Germplasm for Persimmon Breeding. Agronomy $2020,10,1172$. 
21. Polo-Oltra, Á.; Romero, C.; López, I.; Badenes, M.L.; Zuriaga, E. Cost-Effective and Time-Efficient Molecular Assisted Selection for Ppv Resistance in Apricot Based on ParPMC2 Allele-Specific PCR. Agronomy 2020, 10, 1292. [CrossRef]

22. Sapkota, S.; Boatwright, J.L.; Jordan, K.; Boyles, R.; Kresovich, S. Multi-Trait Regressor Stacking Increased Genomic Prediction Accuracy of Sorghum Grain Composition. Agronomy 2020, 10, 1221. [CrossRef] 